\title{
Review of: "Comparison between Analgesia Nociception Index (ANI) and self-reported measures for diagnosing pain in conscious individuals: a systematic review and meta-analysis"
}

\author{
vasilios papaioannou ${ }^{1}$
}

1 Dimocritus University of Thrace

Potential competing interests: The author(s) declared that no potential competing interests exist.

The American Society of Anesthesiologists (ASA) guidelines have recommended that health care providers should adopt standardized and validated tools for evaluation and documentation of pain intensity in different settings, such as the operating room (OR) and the post-anesthesia care unit (PACU), in order to assess efficacy and side effects of pain therapy. In this respect, different technologies have been developed for quantitative pain assessment, such as Analgesia-Nociception Index (ANI), Cardio-vascular Depth of Analgesia (CARDEAN), etc.

Baroni and colleagues in their systematic review and meta-analysis evaluated ANI's accuracy compared to self-reported pain measures, such as numerical rate scale (NRS) and visual analogue scale (VAS), in order to estimate its usefulness in conscious patients. As a result, 16 out of 831 studies were included, whereas a meta-analysis of 9 studies demonstrated a weak negative correlation between ANI and NRS for pain assessment in patients in the post-anesthesia recovery room, or in those submitted to electrical stimulus. According to the authors, the evidence for adopting ANI as an analgesia monitoring tool in conscious individuals is weak compared with NRS. Nevertheless, some patients might be benefited from the use of ANI during procedures or in the immediate post-operative period.

I think the authors have made a significant step towards better understanding of the potential added value of ANI in different groups of patients and in different settings (i.e., post-anesthesia care unit, burn ICU etc). Moreover, as they have clearly illustrated, different anesthetic medications during surgery, as well as level of awareness and potential residual effects of sedatives can add a lot of 'noise' in the signal produced from ANI, particularly upon recovery from anesthesia.

However, the authors should provide a more in-depth discussion about the physiology related to nociception and ANI measurements. The ANI is a non-invasive device that takes an ECG analog output from the patient monitor and displays an average measurement of ANI made over the previous $120 \mathrm{sec}$, based on respiratory influence on the RR interval. It permits a quantitative measurement of HRV, that is the variability of RR in the ECG time series, primarily mediated by parasympathetic and sympathetic outflow from the central nervous system to the sinoatrial node. For this reason and according to published recommendations, a $250 \mathrm{~Hz}$ digitized ECG signal is used for $\mathrm{R}$ wave detection, automatic filtering and RR 
interval computation. HRV is measured using a fast Fourier transformation of the ECG signal that gives rise to two main spectral peaks: high frequency fluctuations corresponding to $0.15-0.5 \mathrm{~Hz}$ and mediated predominately by parasympathetic outflow and low frequency fluctuations $(0.04-0.15 \mathrm{~Hz})$ that reflect both sympathetic and parasympathetic influences on RR time series. When parasympathetic tone is present, each inspiration induces slight tachycardia, corresponding to the respiratory sinus arrhythmia (RSA), with predominance of high frequency periodicities in the spectral pattern of HRV. In case of inadequate antinociception parasympathetic tone decreases, the respiratory influence is dampened and there is a shift of HRV towards the low frequency region.

The ANI monitor uses mathematical analysis for differentiation between sympathetic and parasympathetic effects and keeps only the last variations, leading to the RRHF series. By detecting local minima and maxima of respiratory influence in the RR intervals and computing the area under the filtered RRHF series curve values in 4 subsequent $16 \mathrm{sec}$ sub-windows, the ANI monitor provides a 0-100 index as a fraction of the total window surface:

$\mathrm{ANI}=100 *\left(\alpha^{*}\right.$ AUC $\left.\min +\beta\right) / 12.8$, AUC: area under the curve where $\alpha=5.1$ and $\beta=1.2$, in order to keep the coherence between visual effects of respiratory influence upon RR time series and the quantitative assessment of ANI. By moving the $64 \mathrm{sec}$ window after each calculation, the monitor provides continuous measurement of ANI with a $4 \mathrm{sec}$ moving period, giving an acceptable trend of the parameters values. In case of inadequate anti-nociception, parasympathetic tone decreases in relation to sympathetic activity, leading to decreased ANI values.

As has been suggested, the hypnotic drugs chosen for maintaining general anesthesia before arousal at the PACU may play major role in HRV measurements during and after surgery and could explain contradictory results presented previously. Thus, it seems that patients who receive propofol during general anesthesia demonstrate a good correlation between ANI and NRS values post-operatively, whereas halogenated agents and particularly sevoflurane administration is associated with a blunted ANS response after surgery. In addition, both propofol and halogenated agents have been found to affect HRV during surgery, something that might alter ANI measurements upon recovery from anesthesia.

As the authors have described, there is evidence that HRV is linked to emotion whereas the polyvagal theory suggests that the rapid myelinated vagal system allows mammals to inhibit autonomic arousal, for example from pain and augment pain threshold. In this respect, anxiety and stress-related disorders have been found to attenuate such homeostatic mechanisms and decrease HRV both during rest and upon different psychological challenges, such as pain. In addition, experimental research suggests that ventral periaqueductal gray matter (PAG) is responsible for chronic pain alleviation through the vagal nerve, constituting a wide neural network of homeostatic responses, according to the neurovisceral integration theory. The complexity of such interrelations seems to increase even more due to the involvement of higher brain regions in the changes in HRV. Thus, I think that in the context of pain-stress-anxiety that patients are experiencing upon nociception, ANI changes seem to reflect more a state of stress related arousal rather than just pain effects on respiratory sinus arrhythmia. 
Respiratory parameters have not been discussed by the authors, since they can also alter profoundly HRV independent of changes in cardiac autonomic regulation. In this respect, both respiratory frequency and tidal volume can affect the magnitude of RSA [32]. It has been recognized that the HF amplitude decreases with respiratory frequency and increases with tidal volume.

Regarding correlation measures that were used in the majority of recruited studies, the authors have suggested that in the future, accuracy measures or Bland-Altman plot test should be used. I fully support their recommendation but I also consider that a multiparameter approach that combines different autonomic parameters might behave more accurately as an early pain alert system, in conscious patients. Finally, the parallel use of different scoring systems, such as Beck Depression Inventory and Beck Anxiety Inventory could be also used to assess anxiety and stress, since different studies have confirmed that both pain and anxiety are two indissociable aspects of the same phenomenon, triggered by tissue injury. Neverthe-less, pain remains a subjective experience and exhibits extreme individual variability with changing patterns over time. 